\title{
Mid-Infrared Forbidden Lines of NGC 1068, NGC 4151, and NGC 5506
}

\author{
M. A. Greenhouse, ${ }^{1}$ T. L. Hayward, ${ }^{2}$ S. Satyapal, ${ }^{1}$ D. H. Wooden, ${ }^{3}$ J. W. \\ Miles, ${ }^{1}$ F.C. Witteborn, ${ }^{3}$ J.D. Bregman, ${ }^{3}$ C. E. Woodward, ${ }^{4}$ M. A. \\ Malkan, ${ }^{5}$ J. Fischer, ${ }^{6}$ and H. A. Smith ${ }^{1}$ \\ ${ }^{1}$ National Air and Space Museum, Smithsonian Institution, \\ Washington, DC 20560, USA \\ ${ }^{2}$ Department of Astronomy, Cornell University, Ithaca, NY \\ 14853-6801, USA
}

${ }^{3}$ Space Science Division, NASA Ames Research Center, Moffett Field, $C A$ 94035, USA

${ }^{4}$ Department of Physics and Astronomy, University of Wyoming, Laramie, WY 82071, USA

${ }^{5}$ Department of Astronomy, University of California, Los Angeles, CA 90024, USA

${ }^{6}$ Remote Sensing Division, Naval Research Laboratory, Washington, DC 20375, USA

We report near-infrared spectroscopy of three Seyfert galaxies. Velocity resolved spectra covering low excitation-potential transitions of [Ar III] $8.991 \mu \mathrm{m}$, [S IV] $10.514 \mu \mathrm{m}$, and [Ne II] $12.813 \mu \mathrm{m}$ were obtained using the facility mid-infrared array spectrometer (SpectroCam) of the Palomar Observatory Hale 5-m Telescope, and low-resolution spectra covering [Mg v] $5.608 \mu \mathrm{m}$, [Ar II] $6.985 \mu \mathrm{m}$, and [NeVI] $7.642 \mu \mathrm{m}$ were obtained using the faint-object spectrograph (HIFOGS) of the Kuiper Airborne Observatory (KAO).

We find high contrast [ArIII] and [S IV] in each galaxy, and [NeII] in NGC 1068 and NGC 4151 (12.8 $\mu \mathrm{m}$ data were not obtained on NGC 5506). The line profiles are well resolved. In NGC 1068 and NGC 4151, they are fit by singlecomponent Gaussians. However, in NGC 5506, evidence for a broad pedestal is seen in the [ArIII] and [SIV] spectra, similar to the broad $\mathrm{Pa} \beta$ component reported by Blanco et al. (1990) and Rix et al. (1990).

Our airborne spectroscopy of NGC 1068 has revealed two new coronal lines of $\mathrm{Ne}$ and $\mathrm{Mg}$, and unexpectedly bright [ArII] emission. These new data on $[\mathrm{NeVI}]$ and $[\mathrm{Mg} \mathrm{V}]$ double the number of infrared coronal lines detected in NGC 1068. These new airborne and ground-based observations provide the first constraint on the abundance of $\mathrm{Ar}, \mathrm{S}, \mathrm{Ne}$, and $\mathrm{Mg}$ in these nuclei.

Our high-resolution spectra provide new dynamical information on the origin of these lines. A correlation between forbidden-line width and ionization potential or critical density has been observed in optical spectra of several AGN (e.g., Greenhouse et al. 1991) suggesting that high critical-density lines arising from high ions are produced in a dynamical zone interior to circumnuclear starburst regions. However, in both NGC 1068 and NGC 4151, we find no increase 
in line width with increasing critical density among [NeII], [Ar III], and [S IV]. We note that, among the $40 \mathrm{eV}$ ions in both galaxies, the lowest critical-density transition exhibits the greatest line width, in contradiction to conventional wisdom. The $40 \mathrm{eV}$ species exhibit a large dispersion in line width in contrast to the high-ionization lines [Si vi] and [Si VII] (Moorwood \& Oliva 1991) which show identical width and substantial broadening relative to [Ar III] or [NeII]. The dispersion in line broadening we observe among $40 \mathrm{eV}$ ions decreases abruptly among the coronal ions, suggesting that the latter are produced in a dynamically distinct region. This possibility can be tested by high-resolution spectroscopy of intermediate ions such as [Ne VI] with the Infrared Space Observatory (ISO).

We find that the measured [ArIII] flux in NGC 1068 and NGC 4151 is in agreement with the model calculations of Spinoligo \& Malkan (1992; hereafter SM92) suggesting that the argon abundance is approximately normal in the starburst region of these nuclei. However, in NGC 1068, we find that this model underestimates the $[\mathrm{Ar} I \mathrm{II}]$ line by more than two orders of magnitude suggesting that photo-dissociation regions occupy a large volume filling factor within the $1 \mathrm{kpc}$ diameter region observed with the $K A O$. In addition, we find that SM92 underestimates [S IV] by a factor of 7 in NGC 4151, suggesting that sulfur may be over-abundant in this nucleus.

We find that, in NGC 1068, our measured [Ne II] flux is in agreement with SM92. However, this model underestimates [Ne VI] by a factor of 20. If this discrepancy is due to overabundance of neon in the coronal-line emitting region (CLR), then it would provide further circumstantial evidence that the CLR of this nucleus is physically distinct from the starburst region.

\section{References}

Blanco, P. R. 1990, MNRAS, 242, 4P.

Greenhouse, M. A., et al. 1993, ApJS, 88, 23.

Moorwood, A.F.M., \& Oliva, E. 1991, The Messenger, 63, 57.

Rix, H.W., et al. 1990, ApJ, 363, 480.

Spinoglio, L., \& Malkan, M. A. 1992, ApJ, 318, 370. 